\title{
Clinical correlates of complicated grief among individuals with acute coronary syndromes
}

This article was published in the following Dove Press journal:

Neuropsychiatric Disease and Treatment

7 October 2015

Number of times this article has been viewed

\author{
Stefano Pini' \\ Camilla Gesi' \\ Marianna Abelli' \\ Alessandra Cardini' \\ Lisa Lari' \\ Francesca Felice ${ }^{2}$ \\ Rossella Di Stefano ${ }^{2}$ \\ Gianfranco Mazzotta ${ }^{3}$ \\ Francesco Bovenzi ${ }^{4}$ \\ Daniele Bertoli ${ }^{5}$ \\ Lucia Borelli ${ }^{4}$ \\ Paola Michi' \\ Claudia Oligeri ${ }^{3}$ \\ Alberto Balbarini ${ }^{2}$ \\ Vijaya Manicavasagar ${ }^{6}$ \\ 'Department of Clinical and \\ Experimental Medicine, Psychiatry \\ Sector, University of Pisa, Pisa, \\ ${ }^{2}$ Department of Surgical, Medical, \\ Molecular and Critical Area Pathology, \\ University of Pisa, Pisa, ${ }^{3}$ Unit of \\ Cardiology, Ospedale Sant'Andrea, \\ La Spezia, ${ }^{4}$ Cardio-Respiratory \\ Department, Ospedale Campo di \\ Marte, Lucca, ${ }^{5}$ Unit of Cardiology, \\ Ospedale San Bartolomeo, Sarzana \\ (SP), Italy; 'Black Dog Institute, \\ University of New South Wales, \\ Sydney, NSW, Australia
}

Correspondence: Stefano Pini Department of Clinical and Experimental Medicine, Section of Psychiatry, University of Pisa, via Roma 67, 56I26

Pisa, Italy

Tel +3950 221 9780

Email stefano.pini@med.unipi.it
Objective: The study aimed at exploring bereavement and complicated grief (CG) symptoms among subjects without a history of coronary heart disease (CHD) at the time of a first acute coronary syndrome (ACS) and to evaluate the relationship of CG symptoms and ACS.

Method: Overall, 149 subjects with ACS (namely, acute myocardial infarct with or without ST-segment elevation or unstable angina), with no previous history of CHD, admitted to three cardiac intensive care units were included and evaluated by the Structured Clinical Interview for Complicated Grief (SCI-CG), Hamilton Depression Rating Scale, Hamilton Anxiety Rating Scale, and the 36-item Short-Form Health Survey (MOS-SF-36).

Results: Of the total sample of 149 subjects with ACS, 118 (79.2\%) met criteria for DSM-5 persistent complex bereavement disorder. Among these, subjects who lost a partner, child, or sibling were older $(P=0.008)$, less likely to be working $(P=0.032)$, and more likely to be suffering from hypertension $(P=0.021)$, returned higher scores on the SCI-CG $(P=0.001)$ and developed the index ACS more frequently between 12 and 48 months after the death than those who lost a parent or another relative ( $P \leq 0.0001)$. The occurrence of ACS $12-48$ months $(P=0.019)$ after the loss was positively correlated with SCI-CG scores. An inverse relationship with SCI-CG scores was observed for patients who experienced ACS more than 48 months after the loss $(P=0.005)$. The SCI-CG scores significantly predicted lower scores on the "general health" domain of MOSSF-36 ( $P=0.030)$, as well as lower scores on "emotional well-being" domain $(P=0.010)$.

Conclusion: A great proportion of subjects with ACS report the loss of a loved one. Among these, the loss of a close relative and the severity of CG symptoms are associated with poorer health status. Our data corroborate previous data indicating a strong relationship between CG symptoms and severe cardiac problems.

Keywords: acute coronary syndrome (ACS), coronary heart disease (CHD), CG symptoms, complicated grief, DSM-5

\section{Introduction}

A growing body of data indicates that $10 \%-20 \%$ of bereaved persons develop a form of inordinate, prolonged grief, variously referred to as complicated grief (CG), prolonged grief disorder, or traumatic grief and it is included among "disorders requiring further research" in Diagnostic and Statistical Manual of Mental Disorders, 5 th edition (DSM-5) under the name of Persistent Complex Bereavement Disorder. ${ }^{1-4}$

Symptoms of CG include yearning and longing for the deceased, with intense sorrow and painful emotions persisting more than 1 year after the loss. Patients show preoccupation with the deceased and with the circumstances of the death and may have recurrent, intrusive images or thoughts of the death, may feel a sense of disbelief and may avoid reminders of the deceased. In addition, subjects with CG may feel that life is meaningless or empty without the deceased and they may desire to die in order to be reunited with the deceased..$^{5-10}$ 
Several studies suggest that CG impacts uniquely on physical and mental health, increasing the risk for suicidal ideation, substance abuse, chronic medical conditions, and impaired global functioning. ${ }^{11-17}$ Specifically, an association between CG symptoms and the risk of developing cardiac problems has been suggested. ${ }^{11}$ However, to the best of our knowledge, only one study evaluated CG symptoms among subjects with heart diseases. In this report, poorer physical and mental health outcomes were found among patients who had undergone coronary artery bypass grafts with concurrent CG compared to those without CG. ${ }^{18}$

The purpose of this study is to investigate bereavement among inpatients admitted for an acute coronary syndrome (ACS) with no previous history of coronary heart disease (CHD) and to evaluate whether symptoms of CG may have an impact on ACS outcomes and/or physical and mental health-related quality of life.

\section{Methods}

\section{Sample}

Patients with ACS and no previous history of CHD who were admitted to three cardiac intensive care units (CICUs) were eligible for inclusion if they met the criteria for either acute myocardial infarct with ST-segment elevation or without ST-segment elevation or unstable angina, verified by standard ACS criteria. Additional details about study sample and methods have been published elsewhere..$^{19,20}$

The three units were located in a northwest area of Italy, with an average distance of 40 miles from each other and serving similar geographic catchment areas in terms of sociodemographic characteristics. Exclusion criteria were the presence of neoplastic disease, severe pulmonary, hepatic, or renal insufficiency, infections requiring antibiotics, autoimmune disease, immunosuppressive therapy, severe anemia, and severe degenerative disease of the central nervous system. The study protocol was approved, according to Declaration of Helsinki, by the University of Pisa and ASL-5 ethics committees, and written informed consent was obtained from all patients.

\section{Cardiological evaluation}

Data on patients' demographics, medical history, clinical characteristics, electrocardiographic findings, ACS definition and treatment interventions, as well as in-hospital outcomes were reported in a detailed standardized case record form, adapted from the Blitz study, ${ }^{20}$ by a trained cardiologist in each CICU. The details on symptoms onset, first medical help seeking, and arrival at hospital CICU were collected as soon as patients could be interviewed. Particular care was taken in assessing the timing of arrival at hospital CICU, ECG execution, and reperfusion treatment. Additional items included length of stay in the CICU and overall hospital stay, timing of invasive procedures, and transfer to a tertiary care hospital to undergo coronary angiography and/or revascularization.

\section{Risk factors evaluation}

All subjects were assessed at the time of enrollment for the following measures: resting blood pressure and cardiovascular risk factors, including tobacco smoking, diabetes mellitus, and hypertension. Hypertension was defined as either resting systolic or diastolic blood pressure greater than or equal to 140/90 $\mathrm{mmHg}$ at two different times or being on antihypertensive medications. Diabetes mellitus was defined as a serum fasting glucose level greater than or equal to $7.1 \mathrm{mmol} / \mathrm{L}$ or being on hypoglycemic medications.

\section{Psychiatric assessment}

The severity of CG symptoms was investigated by the Structured Clinical Interview for Complicated Grief (SCI-CG), a 33-item semi-structured interview assessing the presence or absence of symptoms associated with CG, as well as details surrounding the death of a loved one..$^{21,22}$

The presence and severity of current depression were assessed by the 17-item Hamilton Depression Rating Scale (HAM-D). ${ }^{23}$ The HAM-D provides an indication of depression and, over time, a guide to recovery. It is widely used to provide accepted outcome measures for evaluating the severity of depression symptoms in ACS patients. Eight items are scored on a 5 -point scale, ranging from $0=$ not present to $4=$ severe. Nine items are scored from 0 to 2 . Scores of 5 or below define the absence of clinically relevant depression; scores between 6 and 13 indicate mild depression; scores from 14 to 18 indicate moderate depression; and scores of 19 and above indicate severe depression. ${ }^{24}$

The severity of anxiety symptoms was evaluated by means of the Hamilton Anxiety Rating Scale (HAM-A). ${ }^{25}$ The scale comprises 14 items, exploring both psychic and somatic anxiety. Each item is scored on a 5-point scale, ranging from 0 (not present) to 4 (severe), where a total score below 17 indicates mild anxiety, a score between 18 and 24 indicates mild-to-moderate anxiety, and scores from 25 and above indicate moderate-to-severe anxiety.

The 36-item Short-Form Health Survey (MOS-SF-36) ${ }^{26}$ was used to assess general health status. The measure comprises 36 items within the following eight subscales: "physical functioning" (extent to which health limits physical 
activities), "role physical" (extent to which physical health interferes with work or other daily activities), "bodily pain" (intensity of pain and effect on normal work), "general health" (personal evaluation of health, current and outlook), "vitality" (feeling energetic), "social functioning" (extent to which physical health or emotional problems interfere with normal social activities), "role emotional" (extent to which emotional problems interfere with work or other daily activities), and "mental health" (general mental health, including depression, anxiety, behavioral and emotional control, general positive affect). The first four subscales comprise the physical component summary score, while the other four subscales comprise the mental component summary score. Subscale raw scores are standardized and range between 0 and 100, where 0 is the worst and 100 the best possible health status. ${ }^{27}$

\section{Statistical analyses}

Significant differences between subjects who lost a parent/a sibling/a partner and those who lost another relative were evaluated by two-tailed $t$-tests for continuous measures and by $\chi^{2}$-tests for categorical variables.

In order to evaluate the health consequences associated with CG, SCI-CG scores were used to predict the various health outcomes. Continuous outcomes (ie, age at index ACS, the number of days spent in the CICU, and MOS-SF-36 domain scores) were analyzed using a series of multiple regressions. In each of these analyses, health outcomes were regressed on the SCI-CG score while controlling for age, sex, and cardiovascular risk factors (tobacco smoking, diabetes mellitus, and hypertension).

Dichotomous dependent variables (eg, presence/absence of single ACS risk factors) were analyzed by a series of logistic regressions. Each model estimated the effects of SCI-CG score on a single dichotomous health outcome while controlling for age, sex, and cardiovascular risk factors.

Comparisons were considered statistically significant at the 0.05 level $(P \leq 0.05)$. Analyses were conducted using SPSS, version 20 (IBM Corporation, Armonk, NY, USA). ${ }^{28}$

\section{Results}

Overall, 149 subjects with ACS and with no previous history of CHD were recruited. Among them, 118 (79.2\%) had experienced the death of a loved one and met the criteria for DSM-5 persistent complex bereavement disorder. The 118 bereaved included in the analyses did not differ from the rest of total group in terms of sex, age, CHD risk factors, HAM-D,
HAM-A, SCI-CG total scores, or MOS-SF-36 domain scores (data not shown here).

Demographical and clinical characteristics of bereaved subjects dichotomized by relationship to the deceased (partner/child/sibling vs parent/other relatives) are shown in Table 1.

Subjects who lost a partner, a child, or a sibling were older $(P=0.008)$ and less likely to be working $(P=0.032)$ than those without such losses. They showed significantly greater scores on the SCI-CG $(P=0.001)$ and were more likely to have developed the index ACS between 12 and 48 months after the death than those who lost a parent or another relative $(P \leq 0.0001)$. The two groups did not differ on outcomes of current ACS. However, subjects who lost a partner, a child, or a sibling were more likely to have been diagnosed with hypertension $(P=0.021)$ than those who lost a parent or another relative.

Table 2 demonstrates linear and logistic regression models in which SCI-CG score served as predictor of various clinical correlates in the overall sample of bereaved subjects with ACSs.

Controlling for age, sex, and CHD risk factors, mean SCI-CG scores were correlated with the occurrence of the ACS between 12 and 48 months $(P=0.019)$ and inversely correlated with the onset of the ACS more than 48 months after the loss $(P=0.005)$. Moreover, SCI-CG scores significantly predicted lower scores on the "general health" domain of MOS-SF-36 ( $P=0.030)$, as well as lower scores on "emotional well-being" domain $(P=0.010)$ controlling for age, sex, HAM-D and HAM-A scores.

\section{Discussion}

This study aimed at exploring bereavement and CG symptoms among subjects with no history of CHD at the time of a first ACS and at evaluating the impact of CG symptoms on ACS correlates and health status. We found that the majority (79.2\%) of individuals with a recent ACS had experienced the death of a loved one and had presented symptoms of persistent complex bereavement disorder. Interestingly, while bereavement itself was not associated with specific sociodemographic and clinical characteristics, some differences emerged with respect to association between the loss and some clinical variables preceding ACS. In fact, the loss of a close relative (namely, a partner, a child, or a sibling) was associated with the presence of hypertension and with worse health status with respect to the loss of a parent or other relative. Moreover, while the majority of subjects, independently from the relationship to the deceased, had 
Table I Demographic and clinical characteristics of I I 8 individuals with no previous history of CHD admitted for index ACS to three $\mathrm{CICUs}$ dichotomized by relationship to the deceased

\begin{tabular}{|c|c|c|c|c|}
\hline Characteristic & $\begin{array}{l}\text { Lost partner/child/ } \\
\text { sibling }(n=23)\end{array}$ & $\begin{array}{l}\text { Lost parent/ } \\
\text { other }(n=95)\end{array}$ & $\chi / t$ & $P$-value \\
\hline $\mathrm{Age}^{\mathrm{c}}$ & $64.1(9.4)$ & $57.7(9.8)$ & -2.690 & 0.008 \\
\hline Female sex ${ }^{b}$ & $4(17.4)$ & $17(17.9)$ & - & 1 \\
\hline Years of education ${ }^{c}$ & $9.5(4.3)$ & $10.9(3.7)$ & 1.458 & 0.148 \\
\hline Working & $9(39.1)$ & $62(65.3)$ & 5.277 & 0.032 \\
\hline Time between death and ACS $<12$ months $s^{\mathrm{a}, \mathrm{b}}$ & I (4.3) & $8(8.4)$ & - & 0.686 \\
\hline $12-48$ months $^{\mathrm{a}, \mathrm{b}}$ & $5(21.7)$ & $3(3.2)$ & - & 0.007 \\
\hline More than 48 months $^{\mathrm{a}, \mathrm{b}}$ & $17(73.9)$ & $83(87.4)$ & - & 0.100 \\
\hline SCl-CG score ${ }^{c}$ & $47.7(13.9)$ & $35.7(6.9)$ & -3.978 & 0.001 \\
\hline HAM-D score ${ }^{c}$ & $5.3(4.3)$ & $4.9(5.0)$ & -0.539 & 0.591 \\
\hline HAM-A score ${ }^{c}$ & $5.8(4.0)$ & $5.1(5.0)$ & -0.615 & 0.540 \\
\hline Diabetes $^{\mathrm{b}}$ & $6(26.1)$ & II (II.6) & 3.161 & 0.097 \\
\hline Smoking ${ }^{\mathrm{b}}$ & $14(4.3)$ & $56(55.8)$ & 0.013 & I \\
\hline Hypertension ${ }^{\mathrm{b}}$ & $17(73.9)$ & $44(46.3)$ & 5.648 & 0.021 \\
\hline STEMI ${ }^{b}$ & $16(69.6)$ & $63(66.3)$ & 0.010 & 1 \\
\hline NSTEMI ${ }^{b}$ & $7(30.4)$ & $24(25.3)$ & 0.177 & 0.793 \\
\hline Unstable angina ${ }^{b}$ & $0(0)$ & $5(5.3)$ & - & $0.58 \mathrm{I}$ \\
\hline Days spent in the $\mathrm{CICU}^{c}$ & $3.8(2.6)$ & $5.4(5.4)$ & 1.351 & 0.179 \\
\hline SF-36 physical functioning & $45.0(4 \mid .2)$ & $46.9(32.9)$ & 0.205 & 0.839 \\
\hline Role physical ${ }^{\mathrm{c}}$ & $64.1(46.1)$ & $75.3(4 \mid .6)$ & -0.845 & 0.400 \\
\hline Role emotional ${ }^{c}$ & $64.1(46.1)$ & $75.3(4 \mid .6)$ & 0.855 & 0.396 \\
\hline Energy-fatigue $^{c}$ & $52.6(15.6)$ & $58.8(17.0)$ & 1.582 & 0.116 \\
\hline Emotional well-being ${ }^{c}$ & $60.9(15.6)$ & $66.9(17.2)$ & 1.523 & 0.130 \\
\hline Social functioning ${ }^{c}$ & $64.7(27.1)$ & $71.4(27.5)$ & 1.064 & 0.289 \\
\hline Pain $^{c}$ & $78.3(19.9)$ & $71.5(23.9)$ & -1.260 & 0.210 \\
\hline General health ${ }^{c}$ & $51.0(19.6)$ & $57.8(16.4)$ & 1.709 & 0.090 \\
\hline
\end{tabular}

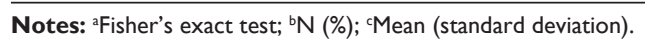

Abbreviations: CHD, coronary heart disease; ACS, acute coronary syndrome; $\mathrm{ClCU}$, cardiac intensive care unit; SCl-CG, structured clinical interview for complicated grief; HAM-D, Hamilton depression rating scale; HAM-A, Hamilton anxiety rating scale; STEMI, with ST-segment elevation; NSTEMI, without ST-segment elevation.

developed the index ACS more than 48 months after the death, a significantly higher proportion (approximately one in five) of individuals who lost a close relative had developed the index ACS between 12 and 48 months after the death. In addition, they had higher levels of CG symptoms than subjects who lost a parent or other relative. The fact that that the nature and quality of the relationship between the subjects and their deceased relative were not measured limits the interpretation of these associations. However, the finding of higher levels of CG among closely related people as partner, siblings, and children is consistent with several data from the literature. The loss of a child is among the most distressing experiences in life. ${ }^{29,30}$ Bereaved parents have been recently found to have more severe CG than those who have suffered another loss. ${ }^{22}$ In two previous investigations conducted in the general population, the loss of a spouse or a child was shown to heighten the risk of developing CG..$^{31,32}$ Moreover, in another population-based study of bereaved adults who had lost a brother or a sister to cancer, the majority of subjects had not worked through their grief over the sibling's death after $2-9$ years. ${ }^{33}$
Another important finding is that, in the overall sample of bereaved subjects, after controlling for effect of age, sex, and ACS risk factors, severity of CG symptoms was positively correlated with the occurrence of the ACS between 12 and 48 months, and inversely correlated with the occurrence of the ACS later than 48 months after the death of a closely related person.

Our results are commensurate to those of Ghesquiere et $a 1^{18}$ the only previous study, to our knowledge, to examine CG in a population with coronary artery disease. The authors found that $90 \%$ of depressed participants had experienced the death of a loved one. A positive CG-screen was associated with more severe depression, poorer mental health related quality of life, lower social support, higher tobacco use, increased use of antidepressant medication, and poorer disease-specific physical functioning. Adding to previous longitudinal data showing that CG symptoms might be chronologically related to the development of cardiac problems, ${ }^{11}$ our findings seem to indicate a "time frame" in which the severity of CG is more likely to be associated to the index ACS. Interestingly, 12 months is precisely the 
Table 2 Unstandardized regression coefficients and ORs for predictors of mental and physical health outcomes for bereaved persons with index ACS

\begin{tabular}{|c|c|c|c|c|c|c|}
\hline \multirow[t]{3}{*}{ Dependent variable } & \multicolumn{6}{|c|}{ Independent variable: SCI-CG score } \\
\hline & \multicolumn{6}{|c|}{ Model parameter estimates } \\
\hline & B & SE & OR & $95 \% \mathrm{Cl}$ & $\begin{array}{l}\text { Goodness } \\
\text { of fit }\left(\chi^{2} / R^{2}\right)\end{array}$ & Significance \\
\hline Diabetes $^{a}$ & -0.014 & 0.033 & 0.99 & $0.93-1.05$ & $1.606^{\mathrm{b}}$ & 0.682 \\
\hline Smoking ${ }^{\mathrm{a}}$ & 0.040 & 0.025 & 1.04 & $0.99-1.09$ & $16.266^{b}$ & 0.112 \\
\hline Hypertension $^{\mathrm{a}}$ & 0.005 & 0.022 & 1.01 & $0.96-1.05$ & $14.723^{\mathrm{b}}$ & 0.817 \\
\hline $\mathrm{APA}^{\mathrm{a}}$ & -0.030 & 0.037 & 0.97 & $0.90-1.04$ & $1.77 I^{b}$ & 0.412 \\
\hline Beta-blockers $^{\mathrm{a}}$ & -0.013 & 0.035 & 0.99 & $0.92-1.06$ & $0.210^{\mathrm{b}}$ & 0.704 \\
\hline $\mathrm{ACE}$-inibitors ${ }^{\mathrm{a}}$ & -0.004 & 0.028 & 0.99 & $0.94-1.05$ & $7.928^{\mathrm{b}}$ & $0.88 I$ \\
\hline Statins $^{a}$ & -0.006 & 0.038 & 0.99 & $0.92-1.07$ & $5.385^{b}$ & 0.867 \\
\hline \multicolumn{7}{|l|}{ Time between death and $\mathrm{ACS}^{c}$} \\
\hline Less than 12 months $^{c}$ & 0.025 & 0.034 & 1.03 & $0.96-1.10$ & $4.863^{\mathrm{b}}$ & 0.457 \\
\hline Between 12 and 48 months ${ }^{c}$ & 0.145 & 0.062 & 1.16 & $|.02-| .3 \mid$ & $10.249^{b}$ & 0.019 \\
\hline More than 48 months $^{c}$ & -0.084 & 0.030 & 0.92 & $0.87-0.98$ & $10.872^{\mathrm{b}}$ & 0.005 \\
\hline Age at index $A C S^{d}$ & 0.102 & 0.104 & - & - & $0.022^{\mathrm{e}}$ & 0.331 \\
\hline Days spent in the $\mathrm{CICU}^{f}$ & -0.035 & 0.054 & - & - & $0.072^{\mathrm{e}}$ & 0.521 \\
\hline SF-36 physical functioningf & 0.044 & 0.350 & - & - & $0.086^{e}$ & 0.901 \\
\hline Role physical $^{f}$ & -0.692 & 0.403 & - & - & $0.044^{\mathrm{e}}$ & 0.088 \\
\hline Role emotionalg ${ }^{g}$ & -0.567 & 0.532 & - & - & $0.267^{e}$ & 0.291 \\
\hline Energy-fatigue ${ }^{g}$ & -0.263 & 0.156 & - & - & $0.286^{\mathrm{e}}$ & 0.094 \\
\hline Emotional well-being ${ }^{g}$ & -0.345 & 0.132 & - & - & $0.500^{\mathrm{e}}$ & 0.010 \\
\hline Social functioning ${ }^{g}$ & -0.211 & 0.254 & - & - & $0.280^{\mathrm{e}}$ & 0.408 \\
\hline Pain $^{\mathrm{h}}$ & -0.017 & $0.24 I$ & - & - & $0.072^{\mathrm{e}}$ & 0.093 \\
\hline General health ${ }^{h}$ & -0.389 & 0.177 & - & - & $0.074^{\mathrm{e}}$ & 0.030 \\
\hline
\end{tabular}

Notes: ${ }^{a} \mathrm{SCl}-\mathrm{CG}$ score, age, sex simultaneously entered as independent variables in the linear regression model. ${ }^{b} \chi^{2}$ values. ${ }^{\mathrm{S} C l} \mathrm{C}-\mathrm{CG}$ score, age, sex, antihypertensive treatment simultaneously entered as independent variables in the linear regression model. ${ }^{\mathrm{S}} \mathrm{Cl}-\mathrm{CG}$ score, age, sex, smoking, beta-blockers use, simultaneously entered as independent variables in the linear regression model. ${ }^{e}{ }^{2}$ values. ${ }^{f} \mathrm{SCl}-\mathrm{CG}$ score, hypertension, diabetes, age, sex, simultaneously entered as independent variables in the linear regression model. ${ }^{\mathrm{SCCl}} \mathrm{CG}$ score, HAM-D, HAM-A, age, sex, simultaneously entered as independent variables in the linear regression model. hSCl-CG score, hypertension, smoking, diabetes, sex, simultaneously entered as independent variables in the linear regression model.

Abbreviations: SE, standard error of mean; ORs, odds ratios; ACS, acute coronary syndrome; APA, anti platelet agent; SCl-CG, structured clinical interview for complicated grief; $\mathrm{CICU}$, cardiac intensive care unit; HAM-D, Hamilton depression rating scale; HAM-A, Hamilton anxiety rating scale.

time lapse that discriminates normal grief from persistent grief. In fact, in the DSM-5, persistent complex bereavement disorder is diagnosed only if at least 12 months (6 months in children) have elapsed since the death of someone with whom the bereaved had a close relationship (Criterion A). ${ }^{4}$ Within this framework, our data suggest that the severity of pathological reaction rather than physiological grief symptoms could have an impact on facilitating the onset of the ACS. Finally, the severity of CG symptoms was found to be significantly associated to poor health status, as evaluated by the MOS-SF-36. The association was significant for emotional well-being, after controlling for both anxiety and depressive symptom severity. This finding corroborates previous data indicating that $\mathrm{CG}$, despite being highly comorbid with depression, may impact on quality of life independently of concomitant depressive symptoms. ${ }^{9,34-36}$ It is also noteworthy that $\mathrm{CG}$ symptoms demonstrated a negative impact on MOS-SF-36 "general health," even when the presence of diabetes and hypertension were controlled for.
Several limitations need to be acknowledged in presenting our findings. First, we included subjects with no previous history of CHD and who were hospitalized at their first ACS, which may reduce the generalizability of our results. Second, the study sample was relatively small, with a small number of patients in the group of subjects who lost a close relative; thus, some comparisons with respect to kinship to the deceased may have been significant with a larger sample. Notwithstanding, it should also be kept in mind that psychiatric assessments of ACS patients, especially in relation to loss events, is not straightforward because of the patient's objective physical status. It is possible that there was not sufficient power to detect meaningful impacts of CG symptoms on some health variables. For example, a previous study found a strong correlation between CG and increased consequent tobacco smoking, ${ }^{37}$ an association that we failed to find in our study. Analogously, potential limited power imposes cautiousness in interpreting results of regression analyses, where multiple suitable explanatory 
variables were used. Third, although depression and anxiety symptoms were investigated, the study lacked a complete psychiatric evaluation, thus our findings could also be related to other, unmeasured confounders, such as other concurrent mental disorders.

Our data suggest a temporal relationship between $\mathrm{CG}$ and the occurrence of the first acute coronary episode. The differential temporal effect of grief in our sample (ie, 12-48 months vs $>48$ months) stimulates further research to develop preventive interventions for cardiovascular patients and investigate potential effects of $\mathrm{CG}$ on endothelial/ vascular parameters connected to occurrence of ACSs.

\section{Acknowledgment}

Financial support for conducting the research was provided by the Fondazione CARISPE, La Spezia, Italy.

\section{Disclosure}

The authors report no conflicts of interest in this work.

\section{References}

1. Lichtenthal WG, Cruess DG, Prigerson HG. A case for establishing complicated grief as a distinct mental disorder in DSM-V. Clin Psychol Rev. 2004;24:637-662.

2. Prigerson HG, Horowitz MJ, Jacobs SC, et al. Prolonged grief disorder: psychometric validation of criteria proposed for DSM-V and ICD-11. PLoS Med. 2009;6(8):e1000122.

3. Shear K, Simon N, Wall M, et al. Complicated grief and related bereavement issues for DSM-5. Depress Anxiety. 2011;28(2):103-117.

4. American Psychiatric Association. Diagnostic and Statistical Manual of Mental Disorders. 5th ed. Washington, DC: American Psychiatric Association; 2013.

5. Horowitz MJ, Siegel B, Holen A, Bonanno GA, Milbrath C, Stinson CH. Diagnostic criteria for complicated grief disorder. Am J Psychiatr. 1997;154:904-910.

6. Shear K, Shair H. Attachment, loss, and complicated grief. Dev Psychobiol. 2005;47:253-267.

7. Shear K, Monk T, Houck P, et al. An attachment-based model of complicated grief including the role of avoidance. Eur Arch Psychiatr Clin Neurosci. 2007;257(8):453-461.

8. Boelen PA, van den Bout J. Complicated grief and uncomplicated grief are distinguishable constructs. Psychiatr Res. 2008;157:311-314.

9. Zisook S, Shear K. Grief and bereavement: what psychiatrists need to know. World Psychiatr. 2009;8(2):67-74.

10. Zisook S, Simon NM, Reynolds CF, et al. Bereavement, complicated grief, and DSM, part 2: complicated grief. J Clin Psychiatr. 2010; 71(8):1097-1098.

11. Prigerson HG, Bierhals AJ, Kasl SV, et al. Traumatic grief as a risk factor for mental and physical morbidity. Am J Psychiatr. 1997;154(5):616-623.

12. Kristensen P, Weisaeth L, Hussain A, Heir T. Prevalence of psychiatric disorders and functional impairment after loss of a family member: a longitudinal study after the 2004 tsunami. Depress Anxiety. 2015;32(1):49-56.

13. Boelen PA, van de Schoot R, van den Hout MA, de Keijser J, van den Bout J. Prolonged grief disorder, depression, and posttraumatic stress disorder are distinguishable syndromes. J Affect Disord. 2010;125(1-3): 374-378.

14. Bonanno GA, Neria Y, Mancini A, Coifman KG, Litz B, Insel B. Is there more to complicated grief than depression and PTSD? J Abnorm Psychol. 2007;116:342-351.
15. Dillen L, Fontaine JR, Verhofstadt-Denève L. Confirming the distinctiveness of complicated grief from depression and anxiety among adolescents. Death Stud. 2009;33:437-461.

16. Kersting A, Krocker K. Prolonged grief as a distinct disorder, specifically affecting female health. Arch Womens Ment Health. 2010;13:27-28.

17. Dell'Osso L, Carmassi C, Rucci P, Ciapparelli A, Conversano C, Marazziti D. Complicated grief and suicidality: the impact of subthreshold mood symptoms. CNS Spectr. 2011;16(1):1-6.

18. Ghesquiere A, Shear MK, Gesi C, et al. Prevalence and correlates of complicated grief in adults who have undergone a coronary artery bypass graft. $J$ Affect Disord. 2012;136(3):381-385.

19. Di Stefano R, Felice F, Pini S, et al. Impact of depression on circulating endothelial progenitor cells in patients with acute coronary syndromes: a pilot study. J Cardiovasc Med. 2014;15(4):353-359.

20. Pini S, Abelli M, Gesi C, et al. Frequency and clinical correlates of bipolar features in acute coronary syndrome patients. Eur Psychiatr. 2014;29(4):253-258.

21. Bui E, Robinaugh D, Mauro C, et al. Development and preliminary psychometric properties for a structured clinical interview for complicated grief. Poster presented at: Proceedings of the 30th Annual Meeting of the International Society for Traumatic Stress Studies; 2014; Miami, FL.

22. Zetumer S, Young I, Shear MK, et al. The impact of losing a child on the clinical presentation of complicated grief. J Affect Disord. 2015;170: $15-21$.

23. Hamilton M. Development of a rating scale for primary depressive illness. Br J Soc Clin Psychol. 1967;6:278-283.

24. Rush AJ, Kraemer HC, Sackeim HA, et al; ACNP task force. Report by the ACNP task force on response and remission in major depressive disorder. Neuropsychopharmacology. 2006;31:1841-1853.

25. Hamilton M. The assessment of anxiety states by rating. Br J Med Psychol. 1959;32:50-55.

26. Ware JE, Snow KK, Kosinski M, Gandek B. SF-36 Health Survey Manual and Interpretation Guide. Boston, MA: The Health Institute, New England Medical Center; 1993.

27. McHorney CA, Ware JE Jr, Raczek AE. The MOS 36-Item Short-Form Health Survey (SF-36): II. Psychometric and clinical tests of validity in measuring physical and mental health constructs. Med Care. 1993;31(3): 247-263.

28. IBM Corporation. IBM SPSS Statistics for Windows, Version 20.0. Armonk, NY: IBM Corporation; 2011.

29. Li J, Laursen TM, Precht DH, Olsen J, Mortensen PB. Hospitalization for mental illness among parents after the death of a child. $N$ Engl $J$ Med. 2005;352:1190-1196.

30. Wijingaards-de Meij L, Stroebe M, Schut H, et al. Patterns of attachment and parents' adjustment to the death of their child. Pers Soc Psychol Bull. 2007;33(4):537-548.

31. Newson RS, Boelen PA, Hek K, Hofman A, Tiemeier H. The prevalence and characteristics of complicated grief in older adults. J Affect Disord. 2011;132(1-2):231-238.

32. Kersting A, Brähler E, Glaesmer H, Wagner B. Prevalence of complicated grief in a representative population-based sample. J Affect Disord. 2011;131(1-3):339-343.

33. Sveen J, Eilegård A, Steineck G, Kreicbergs U. They still grieve-a nationwide follow-up of young adults 2-9 years after losing a sibling to cancer. Psychooncology. 2014;23(6):658-664.

34. Simon NM, Shear K, Thompson EH, et al. The prevalence and correlates of psychiatric comorbidity in individuals with complicated grief. Compr Psychiatr. 2007;48(5):395-399.

35. Stroebe M, Schut H, Stroebe W. Health outcomes of bereavement. Lancet. 2007;370(9603):1960-1973.

36. Boelen PA, Prigerson HG. The influence of symptoms of prolonged grief disorder, depression, and anxiety on quality of life among bereaved adults: a prospective study. Eur Arch Psychiatr Clin Neurosci. 2007;257(8):444-452.

37. Neria Y, Gross R, Litz B, et al. Prevalence and psychological correlates of complicated grief among bereaved adults 2.5-3.5 years after September 11th attacks. J Trauma Stress. 2007;20(3):251-262. 
Neuropsychiatric Disease and Treatment

Dovepress

\section{Publish your work in this journal}

Neuropsychiatric Disease and Treatment is an international, peerreviewed journal of clinical therapeutics and pharmacology focusing on concise rapid reporting of clinical or pre-clinical studies on a range of neuropsychiatric and neurological disorders. This journal is indexed on PubMed Central, the 'PsycINFO' database and CAS,

and is the official journal of The International Neuropsychiatric Association (INA). The manuscript management system is completely online and includes a very quick and fair peer-review system, which is all easy to use. Visit http://www.dovepress.com/testimonials.php to read real quotes from published authors.

Submit your manuscript here: http://www.dovepress.com/neuropsychiatric-disease-and-treatment-journal 